\section{Increasing public venous awareness, graduated compression stockings compli- ance and scientific data collection through open events on golf courses: A feasibility study}

\author{
Sergio Gianesini, ${ }^{1,2}$ Steve Zimmet, ${ }^{3}$ \\ Oscar Bottini, ${ }^{4}$ Diana Neuhardt, ${ }^{5}$ \\ Mark Meissner, ${ }^{6}$ Kate Gibson, ${ }^{7}$ \\ Eva Kalodiki, ${ }^{8}$ Christopher R. \\ Lattimer, ${ }^{8,9}$ Paolo Spath, ${ }^{10}$ \\ Lorenzo Tessari, ${ }^{11}$ \\ Mirko Tessari, ${ }^{1}$ Yung Wei Chi, ${ }^{12}$ \\ Maria Grazia Sibilla, ${ }^{1}$ Erica Menegatti ${ }^{1}$ \\ ${ }^{1}$ Translational Surgery Unit, University \\ of Ferrara, Italy; ${ }^{2}$ USUHS University, \\ Bethesda, MD, USA; ${ }^{3}$ Zimmet Vein \& \\ Dermatology Center, Austin, TX, USA; \\ ${ }^{4}$ Vascular Surgery Department, \\ University of Buenos Aires, Argentina; \\ ${ }^{5}$ Compudiagnostics, Inc., Scottsdale, AZ, \\ USA; ${ }^{6}$ Vascular Surgery Department, \\ University of Washington, Seattle, WA, \\ USA; ${ }^{7}$ Lake Washington Vascular Labs, \\ Bellevue, WA, USA; ${ }^{8}$ Josef Pflug \\ Vascular Lab, Imperial College, West \\ London Vascular \& Interventional \\ Centre, London, UK; ${ }^{9}$ Thrombosis \& \\ Hemostasis Lab, Loyola Medical Centre, \\ Maywood, IL, USA; ${ }^{10}$ Vascular Surgery \\ Department, University of Bologna, \\ Italy; ${ }^{11}$ Bassi Foundation, Trieste, Italy; \\ ${ }^{12}$ University of California, Davis, CA, \\ USA
}

\section{Introduction}

On an international level, public venous awareness is still low. ${ }^{1}$ Campaigns to increase it are needed in order to reduce the clinical and economic burden of venous disease, ${ }^{2,3}$ and in particular of venous thromboembolism, a leading cause of preventable death in industrialized countries. ${ }^{4}$ The center for disease control and prevention declared how adequate communication initiatives represent fundamental opportunities to improving health around the world. ${ }^{5}$ Nevertheless, the rapid growth of countless initiatives has led to the need for a deeper analysis in order to determine the real efficacy of these activities. ${ }^{6,7}$ The aim of the present paper is to evaluate the feasibility of a new format in venous awareness promotion.

\section{Materials and Methods}

Seven international public venous awareness events were developed. These events were twinned with top quality Phlebology meetings of the International Union of Phlebology, American College of Phlebology and Latin American Venous Forum. This social project was called Game Over to Leg Failure, as the acronym GOLF and was endorsed by the Italian Olympics Committee and by the Italian Golf Federation. The vision of the project is the promotion of public venous awareness by means of golf and other pleasant activities at the golf course. All events were open to the public. The same field was transformed into an educational pathway.

All the participants, before walking onto the field, received a venous awareness questionnaire with 18 true/false questions about chronic venous disease issues. This questionnaire was aimed at testing the actual knowledge on venous disease and graduated compression stockings (GCS).

After having walked through the 18 holes and having read all the answers on the banners, golfers and non-golfers were asked to fill out the same questionnaire again, in order to detect the final improvement in their knowledge on the topic.

Each right answer to the 18 questions scored one point, wrong answers and not reported answers were scored 0 . At the field, another set of data collection was related to the effect of an 18-hole walk on lower limb drainage and perceived fatigue following the use of different levels of GCS.

A putting green area of 9 holes was dedicated to venous awareness messages too. These educational messages were on the scorecard of all the players, so that they could take home the educational messages.

The following services were offered complimentary: i) venous ultrasound scanning; ii) cardiology consultation; iii) nutritional consultation; iv) sport-medicine consultation; v) physiatry consultation; vi) physiotheray; vii) postural evaluation; viii) adapted physical activity on the treadmill; ix) adapted physical activity with an innovative Jacket for electrical muscle stimulation training and muscular rehabilitation (miha bodytec $\left.{ }^{T M}\right)$; $\mathrm{x}$ ) capacitive and resistive energy transfer (TECAR) therapy.

A workshop about correct prescribing and use of GCS was offered. All the concepts delivered during the day were associated with scientific paper references. A survey was performed to detect an eventual improvement in terms of compliance to GCS 6 months after the event.
Correspondence: Sergio Gianesini, University of Ferrara, via Aldo Moro 8, 44128 Cona (FE), Italy

E-mail: sergiogianesini@hotmail.com

Conference presentation: International Compression Club (ICC) Meeting, Paris, 2017.

This work is licensed under a Creative Commons Attribution 4.0 License (by-nc 4.0).

(C) Copyright S. Gianesini et al., 2018

Licensee PAGEPress, Italy

Veins and Lymphatics 2018; 7:7631

doi:10.4081/vl.2018.7631

\section{Results}

Among the 7 events, 1684 participants attended the events. Three hundred thirtyfour (334) players filled in the venous awareness questionnaire before starting to play the 18-hole course, thus before reading all the banners. Among these players, 305 participants filled in the questionnaire also at the completion of the course: the average score improved from $11 \pm 2.1$ to $16 \pm 1.3$ $(\mathrm{P}<0.0001)$. In $71 \%$ of the questionnaires the attendees answered that there is no difference among GCS defined in $\mathrm{mmHg}$ or denars. Sixty-two (62) lower limb venous ultrasound scans were performed among the participants. Seven-hundred-thirtyseven (737) attendees stopped at the educational corner dedicated to GCS. Three-hundredfortyfive $(345 ; 46.8 \%)$ subjects among the ones who stopped at the GCS corner were already GCS users, nevertheless $35.9 \%$ $(124 / 345)$ of them presented with a previous wrong GCS prescription in terms of size, $20.9 \%(72 / 345)$ with a wrong class of compression (Figure 1).

At 6 months, 227 subjects of the 345 $(65.8 \%)$ who were already GCS users replied to the survey about the compliance change after the event attendance: $41.8 \%$ $(95 / 227)$ of them reported an improvement after the change in the prescription. Of the remaining 392/737 (53.2\%) attendees who have never used GCS before, $37.8 \%$ (148/392) begun to use them. The reason for beginning using GCS was prolonged standing work in $70.9 \%(105 / 148)$, and chronic venous disease detected just during one of the screenings offered at the venous awareness event in 29.1\% (43/148) (Figure $2)$. In the study population in which lower limb volume was assessed and related to perceived exertion (40 subjects), the use of no hosiery led to a $5 \%$ increase in leg volume $(\mathrm{P}<0.0001), 18-\mathrm{mmHg}$ led to a non- 

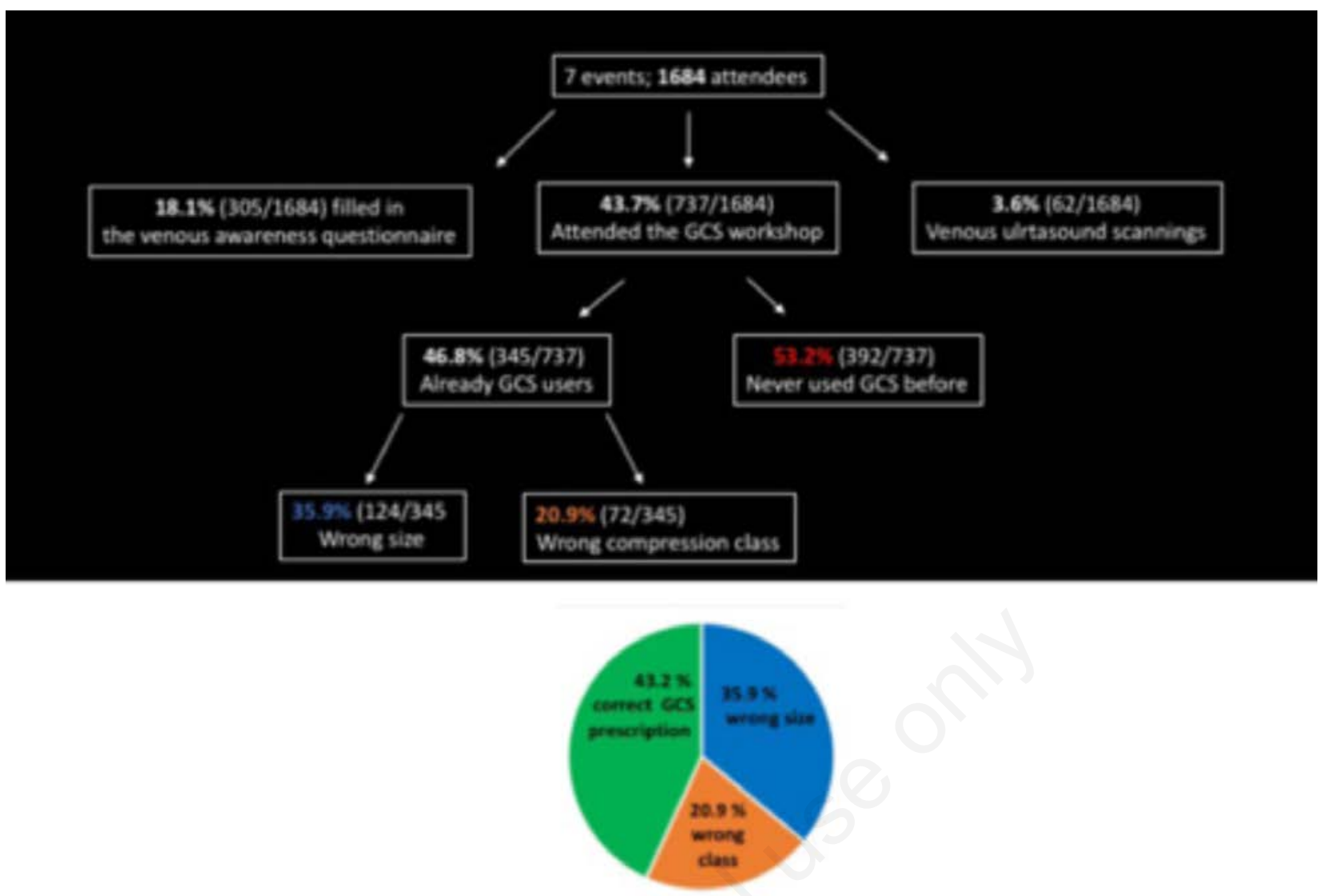

Figure 1. Attendees stopping at the dedicated graduated compression stockings (GCS) workshop.

significant $1 \%$ volume reduction, $23-$ $\mathrm{mmHg}$ to a significantly reduction by $4 \%$. A significant fatigue reduction was reported only using $23-\mathrm{mmHg}$ GCS. ${ }^{8}$

\section{Conclusions}

The present investigation reports an innovative format of public venous awareness. In the United States alone, almost 200-health awareness days, weeks and months are reported on the US national health observances calendar. Nevertheless, the real impact on the global health status of these noble activities is still questionable, due to limiting factors that can influence the final outcome.5This study potentially solves some of the most common weak points of public health awareness initiatives: adequate attendance, attraction of the public, setting feasibility, knowledge improvement assessment and reliability of the educational message. Educational gadgets significantly contributed to continue the health message delivery. An extreme lack of knowledge in the discrimination about
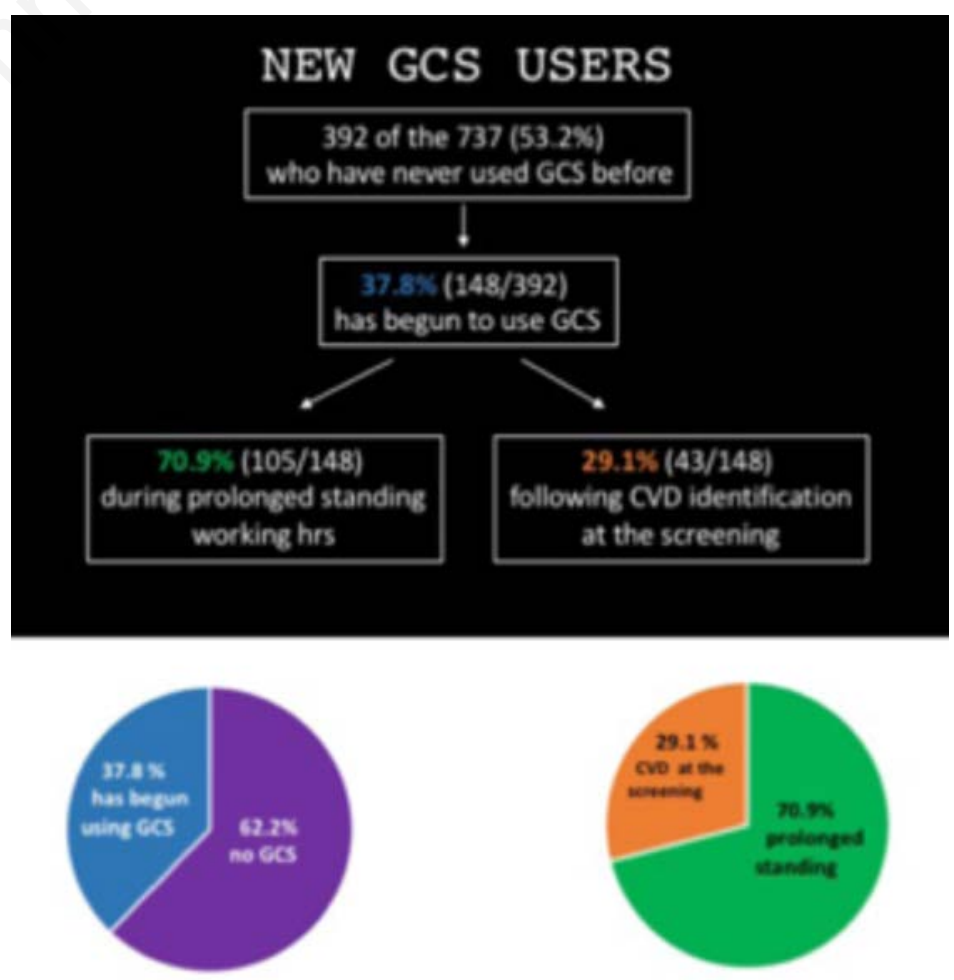

Figure 2. New graduated compression stockings users after the dedicated workshop. 
every day stockings, self-claimed graduated stockings and certified GCS stockings was reported. This finding is in accordance with a previous analysis by Lim, reporting how addressing patients' concerns, providing adequate information and reassurance can positively impact compliance toward GCS. ${ }^{9,10}$

Another major point in public awareness is the promotion of physical activity, in particular for the elderly. ${ }^{11,12}$ The present format involved the general public, older ages included, not only on the health topic, but also on the sport practice.

A wider data collection is ongoing, in order to precisely depict the socio-economic characteristics of the participants.

\section{References}

1. Wendelboe AM. Global public awareness of venous thromboembolism. J Thromb Haemost 2015;13:1365-71.
2. Lefebvre P, Laliberté F, Nutescu EA, et al. All-cause and potentially diseaserelated health care costs associated with venous thromboembolism in commercial, Medicare, and Medicaid beneficiaries. J Manag Care Pharm 2012;18:363-74.

3. Wakefield T. Call to action to prevent venous thromboembolism J Vasc Surg 2009;49:1620-3.

4. Editorial. Thromboembolism: an under appreciated cause of death. Lancet Hematol 2015;2:e393.

5. Bernhardt JM. Communication at the core of effective public health. Am J Public Health 2004;94:2051-53.

6. Purtle J, Roman LA. Health awareness days: sufficient evidence to support the craze? Am J Public Health 2015;105:1061-5.

7. Ayers JW, Westmaas JL, Leas EC, et al. Leveraging big data to improve health awareness campaigns: a novel evaluation of the great american smokeout.
JMIR Public Health Surveill 2016;2:e16.

8. Gianesini S, Tessari M, Menegatti E, et al. Comparison among $18 \mathrm{mmHg}$ and $23 \mathrm{mmHg}$ elastic stockings effect on leg volume and tiredness after golf. Int Angiol 2017;36:129-35.

9. Lim CS, Davies AH. Graduated compression stockings. CMAJ 2014;186:E391-8.

10. Carpentier P, Auvert J-F, Bensedrine S, et al. Compression therapy in everyday life: let the patients have the floor. Veins and Lymphatics 2017;6:6625.

11. King AC, Rejeski WJ, Buchner DM. Physical activity interventions targeting older adults. A critical review and recommendations. Am J Prev Med 1998; 15:316-33.

12. Blair SN, Franklin BA, Jakicic JM, Kibler WB. New vision for health promotion within sports medicine. Am J Health Promot 2003;18:182-5. 